\title{
Expression and correlation of Bcl-2 with pathological grades in human glioma stem cells
}

\author{
BO QIU, YONG WANG, JUN TAO and YUNJIE WANG \\ Department of Neurosurgery, First Hospital of China Medical University, \\ Heping District, Shenyang, Liaoning 110001, P.R. China
}

Received February 24, 2012; Accepted April 12, 2012

DOI: $10.3892 /$ or.2012.1800

\begin{abstract}
The anti-apoptotic gene, B-cell lymphoma-2 (Bcl-2), has been reported to be overexpressed in gliomas and is related to tumor prognosis, suggesting a potential therapeutic target. Additionally, recent studies have demonstrated the existence of brain glioma stem cells (BGSCs) which are tumorigenic, selfrenewable and dominate the biological behavior of gliomas. Currently BGSCs are committed as a new target of glioma therapies. However, few studies have focused on the expression of Bcl-2 in BGSCs. We performed a series of experiments to culture BGSCs from eight clinical specimens, followed by real-time RT-PCR and immunoassays to compare the expression levels of $\mathrm{Bcl}-2$ in BGSCs and their corresponding primary glioma cells (PGCs). The results showed that Bcl-2 mRNA and protein expression levels are higher in BGSCs compared to their counterparts, and the expression levels are related to glioma malignancies. As an anti-apoptotic gene, Bcl-2 assigns immortality characteristics to cells, which coincide with the pivotal biological feature of BGSCs. The experimental results indicated that BGSCs would evade apoptosis for higher Bcl-2 expression, and may interpret the drug resistance of glioma to cytotoxic drugs and other pro-apoptotic agents. New therapies targeting Bcl-2 must induce apoptosis in BGSCs, thus, resulting in treatment or even eradication of glioma.
\end{abstract}

\section{Introduction}

Primary malignant brain tumors are diagnosed in 6-7 per 100,000 persons each year, among which gliomas are the most common and deadliest $(1,2)$. Especially malignant gliomas such as glioblastoma multiformes (GBMs) and anaplastic astrocytomas (AAs), only have a median survival of 14.6 months and an overall survival of only $10 \%$ at 5 years (3). Despite advances in treatment strategies that combine surgery with radiotherapy and chemotherapy, gliomas remain one of the most lethal diseases,

Correspondence to: Dr Yunjie Wang, Department of Neurosurgery, First Hospital of China Medical University, No. 155 North Nanjing Street, Heping District, Shenyang, Liaoning 110001, P.R. China E-mail:wyj024@vip.sina.com

Key words: glioma, brain glioma stem cells, apoptosis, Bcl-2, therapy and become resistant to treatment and inevitably progress $(3,4)$. Upregulation of genes that permit evasion of apoptotic cell death has been observed in high grade gliomas and is a mechanism by which they develop treatment resistance. Proteins of the Bcl-2 (B-cell lymphoma-2) family are thought to contribute to the resistance of glioma cells to anticancer therapy by modulating the apoptotic cascade (4), which suggests that Bcl-2 is a potential target of glioma immunotherapy. However, only a few studies have focused on glioma immunotherapy targeting Bcl-2 (5-7).

In the last decade, the brain glioma stem cells (BGSCs) have been isolated from various types of gliomas and intensively investigated (8-12). BGSCs are now regarded as the initiating cells of glioma and determine the glioma progression, recurrence and resistance to chemotherapy or radiotherapy, by which BGSCs possess 'immortality' and maintain or promote the glioma cell populations $(8-10,13)$. Therefore BGSCs represent an essential cellular target for glioma therapy as they are postulated to be the tumorigenic cells and responsible for therapeutic resistance $(14,15)$. According to existing literature, we hypothesized that $\mathrm{Bcl}-2$ expresses and plays an anti-apoptotic role in BGSCs so as to retain them 'immortal' and resistant to therapies. Thus we carried out a series of assays to isolate and identify BGSCs from clinical glioma specimens and determined expression levels of Bcl-2. The mRNA and protein expression levels were also compared between BGSCs and their corresponding primary glioma cells (PGCs). These experimental data may offer evidence to support the hypothesis and help to better understand the BGSCs theory and glioma biology.

\section{Materials and methods}

Tissue collection and grading. All samples were obtained from 8 adult glioma patients within $30 \mathrm{~min}$ after surgical resection. Each sample was classified by type and grade at China Medical University by experienced chief neuropathologists according to diagnostic criteria of World Health Organization. Eight samples consisted of 2 primary GBMs, 1 recurrent anaplastic oligodendroglioma (AO) and 5 AAs. The protocol of this research was approved by the Institutional Review Boards of the First Clinical Hospital, China Medical University.

Culture of PGCs and tumor spheres. Glioma samples were processed as described previously (9-11,16-18), with minor 
modifications. In brief, tumor samples were washed, acutely minced in PBS and subjected to enzymatic dissociation, then passed through a series of cell strainers, as reported by Singh et al (11), Reynolds and Weiss (19), and Hemmati et al (20). Tumor cells were resuspended in Dulbecco's modified Eagle's medium (DMEM)/F12 (Gibco, Carlsbad, CA, USA) medium containing $15 \%$ fetal bovine serum (FBS, Hyclone, Logan, UT, USA) as growth medium at a density of $2 \times 10^{5}$ live cells $/ \mathrm{ml}$. When glioma cells attached and grew as monolayer in culture flasks for 24-72 h, one flask of these cells was continued to be passaged as PGCs in the same medium, other flasks of the monolayer-growing glioma cells were switched into serum-free DMEM/F-12 (Gibco) supplemented with B-27 (Invitrogen, Carlsbad, CA, USA), $20 \mathrm{ng} / \mathrm{ml}$ recombined human epidermal growth factor (rhEGF, Invitrogen), $20 \mathrm{ng} / \mathrm{ml}$ basic recombined human fibroblast growth factor (rhFGF-b, Invitrogen), $100 \mathrm{IU} /$ $\mathrm{ml}$ penicillin $\mathrm{G}$ and $100 \mu \mathrm{g} / \mathrm{ml}$ streptomycin. Fresh rhEGF and rhFGF-b were added each week. Cells were maintained in a standard tissue culture incubator with $5 \% \mathrm{CO}_{2}$ and $100 \%$ relative humidity at $37^{\circ} \mathrm{C}$ and the medium was changed twice a week. When cell spheres appeared, they were passaged by trituration through a fire-narrowed Pasteur pipette and reseeding into fresh serum-free proliferative medium at the same density to form subspheres. To evaluate the self-renewal capacity of tumor spheres, single-cell suspensions were reseeded into 96-well microwell plates by limited dilution at a cell density of 1-2 live cells per well. Each well was fed with fresh stem cell medium every 2 days. The tumor spheres were inspected and imaged under a phase-contrast microscope (IX 70; Olympus, Tokyo, Japan).

Differentiation of tumor spheres. The tumor spheres were harvested and induced to differentiation by culturing on coverslips precoated with poly-L-lysine (Sigma, St. Louis, MO, USA) in DMEM/F-12 (Gibco) medium containing 15\% FBS in individual wells of a 24-well culture plate. FBS-supplemented medium was changed every 2 days, and coverslips were stained 7 days later.

Immunocytochemical analysis of cultured cells. Immunostaining was performed as described previously (21-23). The undifferentiated tumor sphere cells of approximately the fourth passage were collected and plated onto anti-peeling slides (Corning, NY, USA), and incubated in DMEM/F-12 medium containing rhEGF, rhFGF-b and B-27 for $4 \mathrm{~h}$. After adherence to the slides, tumor spheres were fixed in $4 \%$ paraformaldehyde and incubated with primary mouse monoclonal antibody against CD133 (1:200; Abcam, Cambridge, UK) at $4^{\circ} \mathrm{C}$. After 24 h, Cy3-conjugated goat anti-mouse secondary antibody (1:50; Sigma, St. Louis, MO, USA) was added and incubated for $2 \mathrm{~h}$ at room temperature.

A similar procedure was conducted for the differentiated tumor sphere cells. Following 7 days of differentiation induction, the cells were subjected to an immunocytochemical assay to detect lineage-specific markers. The differentiated cells were fixed in $4 \%$ paraformaldehyde, then incubated with primary antibodies against glial fibrillary acidic protein (GFAP; monoclonal rabbit anti-GFAP, 1:200; Chemicon International, Temecula, CA, USA) for glial cells and TU-20 (monoclonal mouse anti- $\beta$ tubulin III isoform, C-terminus, 1:200; Millipore, Billerica, MA,
Table I. Primers used for real-time PCR.

\begin{tabular}{ll}
\hline Bcl-2 sense & 5'-TATCCAATCCTGTGCTGCTATC-3' \\
Bcl-2 antisense & 5'-ACTCTGTGAATCCCGTTTGAA-3' \\
GAPDH sense & 5'-GCACCGTCAAGGCTGAGAAC-3' \\
GAPDH antisense & 5'-TGGTGAAGACGCCAGTGGA-3'
\end{tabular}

USA) for neurons respectively, overnight at $4^{\circ} \mathrm{C}$. Cy3-conjugated secondary antibody (goat anti-rabbit, 1:250; Sigma) and fluorescein isothiocyanate (FITC)-conjugated secondary antibody (goat anti-mouse, 1:250; Sigma) were then added correspondingly and incubated for $2 \mathrm{~h}$ at room temperature.

Cell nuclei were counterstained with 4,6-diamidino-2-phenylindole (DAPI; Invitrogen). In the control samples, primary antibody was replaced by non-specific IgG. Results were visualized under a fluorescence microscope (BX61; Olympus, Tokyo, Japan).

Extraction of total RNA and quantitative RT-PCR. We applied quantitative real-time reverse-transcription polymerase chain reaction (RT-PCR) to quantify the expression of $\mathrm{Bcl}-2$ in both BGSCs and PGCs. Total RNA was isolated from cells by use of an RNAsimple Total RNA kit (Tiangen Biotech, Beijing, China). The real-time RT-PCR was performed on an ABI PRISM 7000 real-time PCR system (Applied Biosystems, Foster City, CA, USA), using an SYBR PrimeScript RT-PCR Two-Step kit (Takara code: DDR063S, Takara, Japan). Briefly, first-strand cDNA was synthesized from $1 \mu \mathrm{g}$ RNA according to the manufacturer's protocol. Real-time quantitative RT-PCR was performed for the candidate genes and for glyceraldehydes-3-phosphate-dehydrogenase (GAPDH) as internal control. Gene specific primers were designed and synthesized by Takara Inc. (Dalian, China) and listed in Table I. The PCR for each gene was carried out in a $20-\mu 1$ reaction mixture containing $2 \mu \mathrm{l}$ cDNA as template. PCR conditions were: $95^{\circ} \mathrm{C}$ for $10 \mathrm{sec}$ followed by 35 cycles of $95^{\circ} \mathrm{C}$ for $5 \mathrm{sec}, 60^{\circ} \mathrm{C}$ for $31 \mathrm{sec}$. The quantitative RT-PCR data were assessed with the ABI PRISM 7000 software. A standard melting-curve cycle was used to check the quality of amplification. For each analysis, results were calculated by the delta-delta $\mathrm{Ct}$ method and normalized by the expression of housekeeping gene GAPDH. Each sample was analyzed in triplicate.

Bcl-2 enzyme-linked immunosorbent assay (ELISA). A Bcl-2 ELISA (R\&D Systems, Minneapolis, MN, USA) analysis was performed according to the manufacturer's instructions. Briefly, BGSCs and PGCs were cultured in the appropriate medium (either serum-free medium or medium supplemented with $15 \%$ FBS) for $48 \mathrm{~h}$, culture supernatants were harvested and centrifugated at $800 \mathrm{x}$ g for $5 \mathrm{~min}$. Cell numbers in each culture was determined, $1 \times 10^{6}$ cells were defined as one unit and levels of Bcl-2 were expressed as $\mu \mathrm{g} / \mathrm{ml} / \mathrm{U}$. ELISA analysis was carried out on 96-well microplates precoated with the appropriate antibodies. Serial dilutions $(200 \mu \mathrm{l})$ of reference standards or samples were then added to each well. After repeated incubation and washing bound antigen was detected using a conjugated secondary antibody and substrate solutions. The optical density (OD) was measured at $450 \mathrm{~nm}$ with a microplate reader. Each 

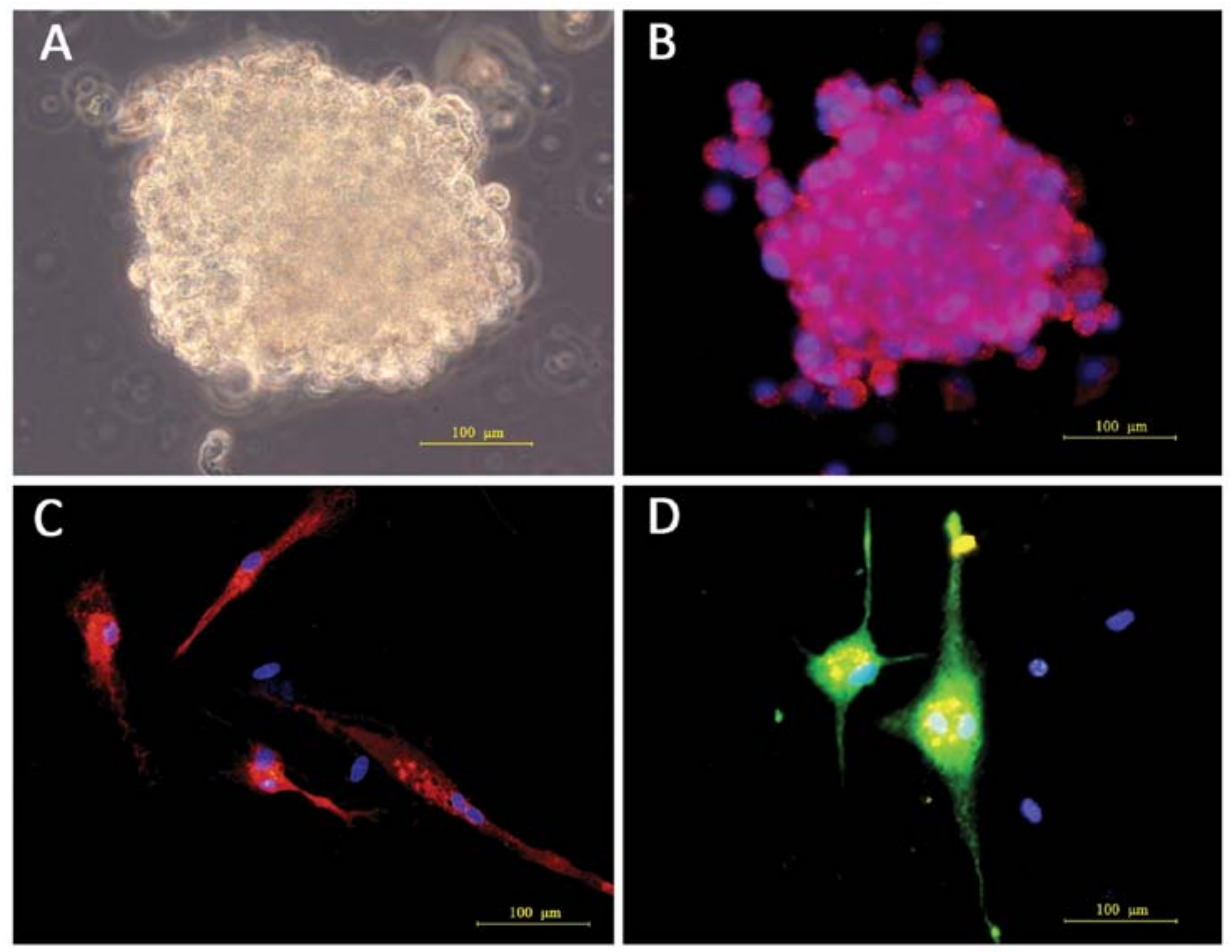

Figure 1. Expression of stem cell, neuronal and glial markers in tumor spheres and differentiated tumor cells. (A) Tumor spheres formed when primary glioma cells were cultured in serum-free stem cell medium. (B) Immunostaining showed tumor sphere cells were CD133-positive (red, magnification x200). Cell nuclei were counterstained with DAPI (blue). (C and D) Immunostaining of differentiated tumor sphere cells for a glial cell marker (GFAP) and a neuronal marker (Tu-20) respectively, cell nuclei are counterstained with DAPI. Tumor spheres differentiated to express GFAP [(C) cy3, red; magnification, x200] and Tu-20 [(D) FITC, green; magnification $\mathrm{x} 200)$.

assay was performed in triplicate, and the concentrations of Bcl-2 were determined and expressed as the mean \pm standard deviation.

Statistical analysis. Data are presented as the mean \pm standard deviation, and analyzed by the two-tailed paired t-test with SigmaPlot 11.0 software (Systat Software, Inc. USA). P<0.05 was considered statistically significanct.

\section{Results}

Glioma cells from clinical specimen can form tumor spheres in vitro. Glioma cells from clinical samples were first cultures as monolayers attached to the bottom of flasks, then the cells were switched into serum-free stem cell medium, which contained EGF, FGF-b and B-27. After 5-7 days of culture, a large numbers of 'neurosphere-like' tumor spheres with spherical or oval appearance were formed, which contained 4-8 cells per sphere. The diameter and cell numbers of the tumor sphere can progressively increase 5-10-fold within 2 weeks by continuing proliferation. After 2 weeks of culture, tumor spheres were photographed under a phase-contrast microscope (Fig. 1A).

Tumor sphere cells show proliferation and self-renewal abilities. Proliferation and self-renewal are critical features of stem cells. In the proliferation assay, tumor spheres were mechanically dissociated into single-cell suspension and passaged at a ratio of 1:2 or 1:3. Cell cleavage was observed 2 days later, and subspheres were formed within 5-7 days. Serial passage revealed that the tumor sphere cells maintained favorable proliferation ability after $\geq 4$ generations. In a limited dilution assay, the single cells from tumor spheres were serially diluted and reseeded in microwells. Counted under a microscope, $>50 \%$ of the single cells in microwells were capable of forming new secondary tumor spheres. Each new secondary sphere contained 10-40 cells, showing the same morphology as the primary sphere. This assay showed that individual stem-like glioma cells from tumor spheres possessed the ability to self-renew and form new subspheres or cell colonies.

Tumor sphere cells are CD133-positive. Immunofluorescence analysis was used to determine if tumor spheres express the committed BGSCs marker CD133. As shown in Fig. 1B, tumor spheres were positive for CD133, indicating that the majority of tumor spheres cells are BGSCs. The nuclei were counterstained, exhibiting marked nuclear atypia.

Tumor sphere cells are multipotent. Upon exposure to differentiation medium, the tumor spheres became flat, and cells began to migrate from the sphere, showing different morphology. Immunocytochemistry staining demonstrated that cells differentiated from tumor sphere were positive for $\beta$-tubulin III, which is a neuron marker (Fig. 1C), or GFAP, which is a glial cell marker (Fg. 1D), consistent with other studies $(9,11,16)$. The results demonstrated that CD133 ${ }^{+}$ tumor sphere cells were multipotent for at least two neural cell types, neurons and glia.

BGSCs express higher levels of Bcl-2 than the PGCs. The realtime RT-PCR revealed that BGSCs express markedly higher 

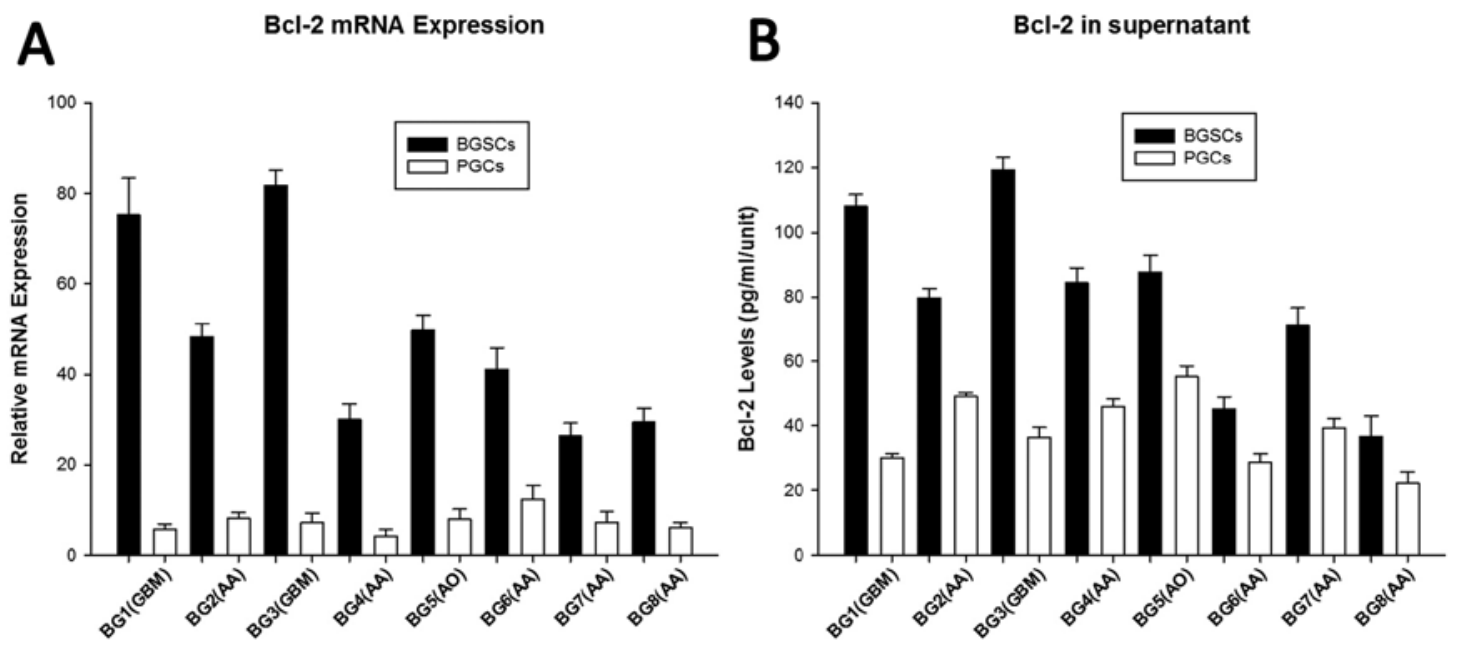

Figure 2. Bcl-2 expression levels in BGSCs and PGCs derived from eight clinical glioma samples. (A) Relative mRNA expression levels of Bcl-2 determined by real-time RT-PCR. (B) ELISA analysis of levels of Bcl-2 antigen in supernatants of cultured BGSCs and PGCs.

levels of Bcl-2 than do their corresponding PGCs, and the elevation was statistically significant using paired t-test analysis $(\mathrm{t}=5.418$ with 7 degrees of freedom, $\mathrm{P}<0.001)$. The results were converted to histograms, as shown in Fig. 2A.

Consistent to the mRNA expression levels, the ELISA results demonstrated that $\mathrm{Bcl}-2$ protein expression levels were significantly higher in BGSCs than in PGCs $(78.94 \pm 28.15 \mathrm{pg} / \mathrm{ml} / \mathrm{U}$ vs. $38.45 \pm 11.23 \mathrm{pg} / \mathrm{ml} / \mathrm{U})$. As shown in Fig. $2 \mathrm{~B}$, the difference is significant ( $\mathrm{P}<0.005$ using paired $\mathrm{t}$-test analysis).

Two grade IV and 6 grade III gliomas were investigated in our experiment, in which BG1 and BG3 are GBMs. We found a tendency for the expression levels of Bcl-2 in BGSCs to be correlated with pathological grade, either the mRNA or the protein levels. The enhanced expression of Bcl-2 seemed relatively higher in GBM-derived BGSCs compared with those from AAs or AO; nevertheless, this trend does not arise among the PGCs from different pathological grades, which is consistent with most literature data that $\mathrm{Bcl}-2$ expression levels were not positively correlated to glioma malignancies (24-28).

\section{Discussion}

The incidence of malignant brain tumors rose steadily over the last quarter of a century in both adults and children (2,29-32). Despite advances in multimodality treatments, the clinical outcomes remain dismal $(1-3,30,32)$. The resistance of gliomas to current treatment protocols is a major concern, and better understanding of glioma biology is a crucial goal of further research to develop novel therapies. Defects in the regulation of apoptotic cell death contribute to the genesis and progression of gliomas. Failure to trigger the cellular suicide program not only predisposes to development of malignancies, but also increases the resistance of gliomas to anticancer drugs and irradiation (33). Apoptosis is a tightly regulated form of programmed cell death involving a series of biochemical events (34-36), in which $\mathrm{Bcl}-2$ gene has been proved to play a critical role in the key events of mitochondrial apoptosis (37-39). The Bcl-2 belongs to a superfamily of genes known to be involved in the regulation of the cell death process, and is predominantly localized in mitochondria that regulates mitochondrial membrane integrity and cytochrome $\mathrm{c}$ release $(40,41)$. As a potent suppressor of apoptosis, alteration of $\mathrm{Bcl}-2$ expression contributes to tumor genesis (42). Bcl-2 gene shows increased expression and upregulation in tumors exhibiting recurrence and progression $(43,44)$, although a correlation between Bcl-2 expression and histological grade in glioma specimens has not been consistently found (24-26,28). Bcl-2 suppresses glioma cell apoptosis induced by chemotherapy and radiotherapy, which results in treatment resistance $(4,35,36,44,45)$. Targeting Bcl-2 may provide a therapeutic benefit by mitigating its anti-apoptotic effects, and it has been reported that inhibition of $\mathrm{Bcl}-2$ with antisense constructs or small-molecule inhibitor, ABT-737, results in glioma cell apoptosis in vitro (6,7). Existing evidence lends support to the strategy of suppressing Bcl-2 signaling as a therapeutic target.

In recent years, BGSCs as another promising therapeutic target were found in glioma investigations $(8,9,11,16)$. BGSCs are defined as a small population of tumor stem cells in gliomas and able to propagate gliomas and promote glioma progression, with the potential for proliferation, self-renewal and differentiation; CD133, a 120-kDa cell-surface protein which is a hallmark of normal human neural precursors, currently serves as a committed marker for the isolation of BGSCs regardless of whether they are solid tumors or glioma cell lines in vitro, although it is shared by other stem cells $(9-11,16,46)$. In vitro experimental data have confirmed that BGSCs are resistant to various adjuvant treatments such as chemotherapy or radiotherapy $(15,17,47,48)$, and the related mechanisms have been investigated accordingly, e.g. activation of DNA damage checkpoint (8) or Notch signaling (49), higher expression of MGMT $\left(\mathrm{O}^{6}\right.$-methylguanine-DNAmethyltransferase) (18), or Akt-induced ABCG2 (ATP-binding cassette sub-family $\mathrm{G}$ member 2) activation (47).

According to the findings above, we hypothesized that $\mathrm{Bcl}-2$ might play a role in BGSCs to evade apoptosis induced by chemotherapy or radiotherapy, because Bcl-2 assigns glioma cells the property of immortality, which is a critical feature of BGSCs to maintain and promote the hierarchal organization of gliomas. Unfortunately, few studies probed into the relationship between Bcl-2 and BGSCs. Thus we conducted a series of assays to deter- 
mine the Bcl-2 expression and evaluate its possible mechanism in BGSCs. After the BGSCs were cultured, isolated and identified from clinical glioma specimens, the expression levels of Bcl-2 in different types of glioma cells were detected by real-time RT-PCR and ELISA. As supposed, Bcl-2 expression is upregulated in BGSCs, either at transcriptional or translational level. Bcl-2 has been found overexpressed in gliomas and considered a responsible factor for glioma resistance to apoptosis-related therapies $(4,33,36,45)$, and our results proved that Bcl-2 is overexpressed in BGSCs either. Furthermore, Bcl-2 expression levels are higher in BGSCs than in PGCs, indicating that BGSCs may have more opportunities than PGCs to evade apoptosis induced by adjuvant treatments. BGSCs have shown resistance in various therapeutic strategies $(8,13,14,17,18)$, although the differentiated glioma cells might respond to chemotherapy or radiotherapy, the progression or relapse of gliomas seem to be inevitable because the BGSCs could not be eradicated. The present experimental data support the hypothesis that $\mathrm{Bcl}-2$ contribute to therapeutic resistance and apoptotic evasion of BGSCs.

Another interesting finding in the present study is that Bcl-2 expression levels seem to be higher in BGSCs from GBMs (grade IV) than in those from AAs or AO (grade III), implying a correlation between Bcl-2 expression and malignancies in BGSCs. As known, it is still controversial whether Bcl-2 expression is related to glioma malignancies. The relative majority of existing data suggested that there is no positive or even a negative correlation between Bcl-2 expression and histological grade (24-26,28,33), but some studies still stated that Bcl-2 expression correlates with poorer prognosis and higher tumor grade $(44,50)$. Theoretically, higher Bcl-2 expression renders glioma cells resistant to anti-tumor treatments, and BGSCs have corresponded with the assumption accordingly in the present investigation. Nevertheless, only 8 cases were included in the present study, further investigation is still required to delineate a definite correlation between Bcl-2 gene and glioma grades.

A few studies have revealed that apoptosis of glioma cells would be induced by inhibition of Bcl-2 (5-7), but whether this induction takes effect on BGSCs is still a question. Based on BGSCs theory, the gliomas would not be cured until the BGSCs are eliminated. Compared to differentiated glioma cells, BGSCs are preferentially equipped with therapeutic resistance mechanisms, so that glioma recurrences after a certain treatment are believed to originate from the surviving BGSCs $(13,51)$. Consequently, BGSCs are an important target for treatment. Eradication of BGSCs should be a judgment criterion in any therapeutic strategy targeting $\mathrm{Bcl}-2$, otherwise progression or recurrence of gliomas seems to be a logically necessary consequence.

In conclusion, we demonstrated that BGSCs display a higher anti-apoptotic gene Bcl-2 expression than PGCs; additionally, Bcl-2 expression levels in BGSCs from grade IV gliomas are higher than those from grade III. Bcl-2 may play an important role in BGSCs maintenance, progression and recurrence. Further understanding of the characterization of BGSC population will shed new light on the underlying biological mechanism of this specific population and, thus, will ultimately lead to the development of more specific therapeutic agents for the treatment of this deadly tumor.

\section{Acknowledgements}

This study was supported by the Foundation of Liaoning Provincial Commission of Science Technology (no. 2007408001-5) and the grant from Chinese National Natural Science Foundation of Youth Science Foundation (no. 81000565).

\section{References}

1. Bondy ML, Scheurer ME, Malmer B, et al: Brain tumor epidemiology: consensus from the Brain Tumor Epidemiology Consortium. Cancer 113: 1953-1968, 2008.

2. Deltour I, Johansen C, Auvinen A, Feychting M, Klaeboe L and Schuz J: Time trends in brain tumor incidence rates in Denmark, Finland, Norway, and Sweden, 1974-2003. J Natl Cancer Inst 101: 1721-1724, 2009.

3. Stupp R, Hegi ME, Mason WP, et al: Effects of radiotherapy with concomitant and adjuvant temozolomide versus radiotherapy alone on survival in glioblastoma in a randomised phase III study: 5-year analysis of the EORTC-NCIC trial. Lancet Oncol 10: 459-466, 2009.

4. Doucette TA and Rao G: B-cell lymphoma-2 promotes malignant progression in glioma. Clin Neurosurg 58: 149-154, 2011.

5. Tagscherer KE, Fassl A, Campos B, et al: Apoptosis-based treatment of glioblastomas with ABT-737, a novel small molecule inhibitor of Bcl-2 family proteins. Oncogene 27: 6646-6656, 2008.

6. Debien E, Hervouet E, Gautier F, Juin P, Vallette FM and Cartron PF: ABT-737 and/or folate reverse the PDGF-induced alterations in the mitochondrial apoptotic pathway in low-grade glioma patients. Clin Epigenetics 2: 369-381, 2011.

7. Jiang Z, Zheng $X$ and Rich KM: Down-regulation of Bcl-2 and Bcl-xL expression with bispecific antisense treatment in glioblastoma cell lines induce cell death. J Neurochem 84: 273-281, 2003.

8. Bao S, Wu Q, McLendon RE, et al: Glioma stem cells promote radioresistance by preferential activation of the DNA damage response. Nature 444: 756-760, 2006.

9. Galli R, Binda E, Orfanelli U, et al: Isolation and characterization of tumorigenic, stem-like neural precursors from human glioblastoma. Cancer Res 64: 7011-7021, 2004.

10. Singh S and Dirks PB: Brain tumor stem cells: identification and concepts. Neurosurg Clin North Am 18: 31-38, 2007.

11. Singh SK, Clarke ID, Terasaki M, et al: Identification of a cancer stem cell in human brain tumors. Cancer Res 63: 5821-5828, 2003.

12. Wang R, Chadalavada K, Wilshire J, et al: Glioblastoma stem-like cells give rise to tumour endothelium. Nature 468 : 829-833, 2010.

13. Venere M, Fine HA, Dirks PB and Rich JN: Cancer stem cells in gliomas: identifying and understanding the apex cell in cancer's hierarchy. Glia 59: 1148-1154, 2011.

14. Dean M, Fojo T and Bates S: Tumour stem cells and drug resistance. Nat Rev Cancer 5: 275-284, 2005.

15. Binello E and Germano IM: Targeting glioma stem cells: a novel framework for brain tumors. Cancer Sci 102: 1958-1966, 2011.

16. Yuan X, Curtin J, Xiong Y, et al: Isolation of cancer stem cells from adult glioblastoma multiforme. Oncogene 23: 9392-9400, 2004.

17. Gilbert CA and Ross AH: Cancer stem cells: cell culture, markers, and targets for new therapies. J Cell Biochem 108: 1031-1038, 2009.

18. Liu G, Yuan X, Zeng Z, et al: Analysis of gene expression and chemoresistance of CD $133^{+}$cancer stem cells in glioblastoma. Mol Cancer 5: 67, 2006.

19. Reynolds BA and Weiss S: Generation of neurons and astrocytes from isolated cells of the adult mammalian central nervous system. Science 255: 1707-1710, 1992.

20. Hemmati HD, Nakano I, Lazareff JA, et al: Cancerous stem cells can arise from pediatric brain tumors. Proc Natl Acad Sci USA 100: 15178-15183, 2003.

21. Wu A, Wiesner S, Xiao J, et al: Expression of MHC I and NK ligands on human $\mathrm{CD} 133^{+}$glioma cells: possible targets of immunotherapy. J Neurooncol 83: 121-131, 2007.

22. Qiu B, Zhang D, Wang C, et al: IL-10 and TGF-beta2 are overexpressed in tumor spheres cultured from human gliomas. Mol Biol Rep 38: 3585-3591, 2011. 
23. Qiu B, Zhang D, Tao J, Wu A and Wang Y: A simplified and modified procedure to culture brain glioma stem cells from clinical specimens. Oncol Lett 3: 50-54, 2012.

24. Yew DT, Wang HH and Zheng DR: Apoptosis in astrocytomas with different grades of malignancy. Acta Neurochir (Wien) 140 341-347, 1998

25. Ambroise MM, Khosla C, Ghosh M, Mallikarjuna VS and Annapurneswari S: The role of immunohistochemistry in predicting behavior of astrocytic tumors. Asian Pac J Cancer Prev 11: 1079-1084, 2010.

26. Takekawa Y, Sawada T and Sakurai I: Expression of apoptosis and its related protein in astrocytic tumors. Brain Tumor Pathol 16: 11-16, 1999.

27. Nakasu S, Nakasu Y, Nioka H, Nakajima M and Handa J: bcl-2 protein expression in tumors of the central nervous system. Acta Neuropathol 88: 520-526, 1994.

28. Newcomb EW, Bhalla SK, Parrish CL, Hayes RL, Cohen H and Miller DC: bcl-2 protein expression in astrocytomas in relation to patient survival and p53 gene status. Acta Neuropathol 94: 369-375, 1997.

29. Jukich PJ, McCarthy BJ, Surawicz TS, Freels S and Davis FG: Trends in incidence of primary brain tumors in the United States, 1985-1994. Neurooncology 3: 141-151, 2001.

30. Deorah S, Lynch CF, Sibenaller ZA and Ryken TC: Trends in brain cancer incidence and survival in the United States: Surveillance, Epidemiology, and End Results Program, 1973 to 2001. Neurosurg Focus 20: E1, 2006.

31. Pirouzmand F and Sadanand V: The incidence trends of primary brain tumors in Saskatchewan from 1970 to 2001. Can J Neuro Sci 34: 181-186, 2007.

32. Hess KR, Broglio KR and Bondy ML: Adult glioma incidence trends in the United States, 1977-2000. Cancer 101: 2293-2299, 2004.

33. Angileri FF, Aguennouz M, Conti A, et al: Nuclear factor-kappaB activation and differential expression of survivin and Bcl-2 in human grade 2-4 astrocytomas. Cancer 112: 2258-2266, 2008.

34. Agostini M, Tucci P and Melino G: Cell death pathology: perspective for human diseases. Biochem Biophys Res Commun 414 451-455, 2011.

35. Steinbach JP and Weller M: Apoptosis in gliomas: molecular mechanisms and therapeutic implications. J Neurooncol 70 247-256, 2004

36. Bogler O and Weller M: Apoptosis in gliomas, and its role in their current and future treatment. Front Biosci 7: E339-E353, 2002 .
37. Green DR and Reed JC: Mitochondria and apoptosis. Science 281: 1309-1312, 1998.

38. Malla R, Gopinath S, Alapati K, et al: Downregulation of uPAR and cathepsin $\mathrm{B}$ induces apoptosis via regulation of $\mathrm{Bcl}-2$ and $\mathrm{Bax}$ and inhibition of the PI3K/Akt pathway in gliomas. PLoS One 5: e13731, 2010

39. Brunelle JK and Letai A: Control of mitochondrial apoptosis by the Bcl-2 family. J Cell Sci 122: 437-441, 2009.

40. Adams JM and Cory S: Life-or-death decisions by the Bcl-2 protein family. Trends Biochem Sci 26: 61-66, 2001.

41. Adams JM and Cory S: The Bcl-2 protein family: arbiters of cell survival. Science 281: 1322-1326, 1998.

42. Yip KW and Reed JC: Bcl-2 family proteins and cancer. Oncogene 27: 6398-6406, 2008.

43. Bruggers CS, Fults D, Perkins SL, Coffin CM and Carroll WL: Coexpression of genes involved in apoptosis in central nervous system neoplasms. J Pediatr Hematol Oncol 21: 19-25, 1999.

44. Strik H, Deininger M, Streffer J, et al: Bcl-2 family protein expression in initial and recurrent glioblastomas: modulation by radiochemotherapy. J Neurol Neurosurg Psychiatry 67: 763-768, 1999.

45. Fels $\mathrm{C}$, Schafer $\mathrm{C}$, Huppe $\mathrm{B}$, et al: $\mathrm{Bcl}-2$ expression in higher-grade human glioma: a clinical and experimental study. J Neurooncol 48: 207-216, 2000

46. Beier D, Hau P, Proescholdt M, et al: CD133(+) and CD133(-) glioblastoma-derived cancer stem cells show differential growth characteristics and molecular profiles. Cancer Res 67: 4010-4015, 2007.

47. Bleau AM, Hambardzumyan D, Ozawa T, et al: PTEN/PI3K/Akt pathway regulates the side population phenotype and ABCG2 activity in glioma tumor stem-like cells. Cell Stem Cell 4: 226-235, 2009.

48. Shervington A and Lu C: Expression of multidrug resistance genes in normal and cancer stem cells. Cancer Invest 26: 535-542, 2008

49. Wang J, Wakeman TP, Lathia JD, et al: Notch promotes radioresistance of glioma stem cells. Stem Cells 28: 17-28, 2010.

50. Weller M, Malipiero U, Aguzzi A, Reed JC and Fontana A: Protooncogene bcl-2 gene transfer abrogates Fas/APO-1 antibody-mediated apoptosis of human malignant glioma cells and confers resistance to chemotherapeutic drugs and therapeutic irradiation. J Clin Invest 95: 2633-2643, 1995.

51. Lamszus K and Gunther HS: Glioma stem cells as a target for treatment. Target Oncol 5: 211-215, 2010. 